Service social

\title{
Pour une éthique de la différence en travail social
}

\section{Ernst Jouthe}

Volume 42, numéro 3, 1993

\section{Perspectives théoriques}

URI : https://id.erudit.org/iderudit/706628ar

DOI : https://doi.org/10.7202/706628ar

Aller au sommaire du numéro

\section{Éditeur(s)}

École de service social de l'Université Laval

ISSN

1708-1734 (numérique)

Découvrir la revue

Citer cet article

Jouthe, E. (1993). Pour une éthique de la différence en travail social. Service social, 42(3), 7-20. https://doi.org/10.7202/706628ar

\section{Résumé de l'article}

Cet article se présente comme une contribution théorique au renouveau de la question éthique dans le monde d'aujourd'hui, en particulier dans la recherche en travail social. Cette réflexion se fait en relation avec les enjeux et défis que pose la diversité de plus en plus marquée de la société québécoise. L'auteur propose un cadre de référence pour négocier de façon coopérative les différences incontournables entre les individus, les groupes et les communautés. L'une des voies de cette négociation consiste dans un dialogue intercompréhensif entre des sujets sociaux différents, à la fois irréductibles et fragiles dans leur identité personnelle et socioculturelle. La tâche principale des intervenantes et intervenants sociaux, toujours à la croisée des chemins, consiste alors à tisser des rapports de reconnaissance symétriques, basés sur le respect des droits et des valeurs de tous dans une société de plus en plus multiraciale, multiethnique et multiculturelle. 


\section{$A \quad R$ T I C L E E S}

\section{Pour une éthique de la différence en travail social}

Ernst JOUTHE

Professeur

Département de travail social, UQAM

\section{INTRODUCTION}

Il faut voir le présent article comme une contribution théorique à la réflexion en cours sur l'éthique ${ }^{1}$ en travail social, en relation avec une problématique très actuelle qui interpelle chercheurs, formateurs, praticiens de l'intervention sociale, et qui consiste dans la difficulté de faire face aux manifestations de plus en plus dramatiques des antagonismes interraciaux, interethniques et interculturels dans le monde, en particulier dans la société québécoise. Il s'agit de situer cette problématique dans une perspective éthico-politique, sous l'éclairage du paradigme de l'unité de l'identité et de la différence.

Notre époque, caractérisée par l'individualisme narcissique, la consommation excessive des biens matériels selon les lois du marché, les inégalités sociales de plus en plus criantes entre riches et pauvres, est aussi celle du chambardement des idéologies et de la crise des valeurs. D'où I'actualité de la question éthique pour tous ceux et toutes celles qui sont confrontés dans la pratique quotidienne aux problématiques émergeant 
dans les sociétés en mutations socioculturelles en cette fin de siècle troublante.

Parmi les documents récents qui attestent l'actualité de la question éthique, signalons le dossier spécial de la revue Relations (1991) intitulé "L'incontournable éthique " et le dossier spécial de la revue Service social (1991) sur "L'éthique et l'intervention sociale ». Malgré leurs angles d'approche différents, les deux dossiers convergent de façon étonnante vers le même constat: il y a un besoin de dépasser aussi bien les codes de déontologie et de morale professionnelle que les approches "réductionniste", " utilitariste " et "légaliste», qui dominent le champ de l'éthique appliquée en Amérique du Nord, pour élever la réflexion éthique au niveau des enjeux collectifs de la société pluraliste et l'orienter dans le sens d'une lecture "critique» de l'expérience vécue dans le monde.

La réflexion éthique $s^{\prime}$ impose en effet partout où le travail social comme profession et comme discipline doit se pencher sur les questions du sens de la vie dans le monde d'aujourd'hui. À titre d'illustration, signalons quelques ouvrages en anglais où la question éthique est abordée spécifiquement sous l'angle du travail social. Dans Social Work Processes (Compton et Galaway, 1975) les trois sections regroupées dans le chapitre 3, "Values in social work practice», sont principalement axées sur des considérations déontologiques très près du "Code d'éthique " de la NASW (National Association of Social Workers) et en relation avec le casework. Le livre Ethical Dilemmas in Social Work Practice (Rhodes, 1986) est à la fois plus récent et plus explicite sur les liens incontournables entre éthique et politique. Dans un article intitulé «What Do We Know About Social Workers' Ethics? ", John Cossom (1992) analyse les raisons de l'attention croissante donnée à l'éthique et au comportement éthique en service social contemporain. Il examine les conclusions de la recherche empirique menée sur l'éthique des travailleurs sociaux, surtout de 1980 à nos jours.

Dans le contexte francophone européen, signalons les actes d'un colloque spécial organisé à Dijon les 9,10 et 11 mars 1988, par la Fédération nationale des Comités d'entente et de liaison des Centres de formation des travailleurs sociaux, publiés sous le titre La théorie et le savoir dans l'acte du praticien. La question éthique dans le champ social et éducatif. Il s'agissait de réaliser en laboratoire, sous forme de journées d'étude, une réflexion multidisciplinaire sur les questions essentielles, y compris les questions philosophiques, auxquelles les praticiens du social sont confrontés dans l'exercice quotidien de leur profession. Le colloque $s^{\prime}$ est terminé sur des questions ouvertes, mais aussi sur des pistes de recherche très stimulantes pour ceux qui veulent orienter la réflexion sur la question éthique vers une perspective plus large que celle des codes de déontologie professionnelle. 
Parmi les pistes de recherche possibles pour aborder la question éthique, nous proposons une réflexion sur le travail de la différence dans la transformation des identités individuelles et collectives. "II n'est pas un seul homme qui diffère d'un autre plus qu'il ne diffère de lui-même dans le cours du temps» (Pascal). Il n'est pas un seul pays, pourrait-on traduire, qui diffère $d^{\prime}$ 'un autre plus que de lui-même dans le cours du temps. Cette réflexion sur le travail de la différence exige au départ une mise au point sur le concept de différence.

\section{PARADIGME DE L'UNITÉ DE L'IDENTITÉ ET DE LA DIFFÉRENCE}

Pour saisir la pleine signification du concept de différence, il faut dépasser les expressions usuelles - et usées! - telles que " respecter les différences ", "écouter la différence ", "vive la différence », etc., pour s'élever au niveau du paradigme de l'unité de l'identité et de la différence. Celui-ci consiste à « trouver l'identité réelle sous la différence et la contradiction apparentes, et la différence substantielle sous I'apparente identité » (Gramsci, 1975 : 2268, trad. EJ). Il s'agit là d'une qualité essentielle à l'intervention sociale, laquelle implique une capacité de distanciation permettant d'éviter l'auto-illusion de nos représentations des choses familières: "les choses qu'on sait comme ça, en général, précisément parce qu'elles sont bien connues et familières, ne sont pas connues» (Hegel, 1991 : 47).

Ce paradigme, sous sa formulation abstraite, recèle une puissance heuristique susceptible d'orienter la recherche et l'intervention sur des pistes concrètes; car " rechercher de manière critique ce qui est semblable dans la différence apparente et ce qui est différent et opposé dans I'uniformité apparente, organiser et relier étroitement ce qui est semblable", tout cela peut se faire d'une façon telle que "cette organisation et ce lien apparaissent comme une nécessité pratique ». Ce travail critique implique concrètement " une unité organique entre théorie et pratique, entre couches intellectuelles et masses, entre gouvernants et gouvernés " (Gramsci, 1983: 449).

L'élément essentiel pour organiser et relier étroitement ce qui est semblable, c'est l'information. Bateson définit l'information comme "la différence qui fait la différence», la «nouvelle» de la différence. Pour créer les nouvelles de la différence, il faut deux entités (réelles ou imaginaires) telles que la différence qui existe entre elles puisse appartenir en propre à leur relation mutuelle (Bateson, 1984: 74). Une différence est ainsi une relation dialectique entre deux aspects contraires, voire opposés d'une même réalité. Ce qui fait la richesse de cette réalité, c'est précisément le fait qu'elle se présente comme une unité dans la diversité, 
une union qui différencie. Tout objet, tout événement ou différence dans ce qu'on appelle le «monde extérieur » peut devenir une source d'information à condition d'être incorporé dans un circuit comprenant un réseau approprié d'éléments souples, où il peut provoquer des changements (Bateson, 1984: 117).

La différence, source d'information, provocatrice de changements, fait toujours partie d'un contexte, d'une situation d'interface, qui lui donne son sens. La description et l'analyse seront d'autant plus stimulantes que l'on prendra soin de relever, comme sur une carte, les plus petites différences présentes dans les aspects multiples de cette situation. L'interprétation de ces différences n'est guère chose facile, car elle est influencée, ici et maintenant, par I'histoire personnelle, familiale et sociale de chaque individu, par son éducation, sa formation et ses conditions de vie, par la place qu'il occupe dans la société civile et politique, et par son degré d'intériorisation des idéologies et des normes de la culture dominante. Toutes ces influences entraînent parfois une attitude rigide à l'égard des différences: au lieu de les situer dans leur contexte, on les en isole, on en fait des abstractions, des étiquettes commodes pour nommer les choses et les personnes qui nous entourent. Ces étiquettes sont tellement confortables qu'on leur attribue une vertu qu'elles $n^{\prime}$ ont pas: elles sont prises pour la réalité elle-même, alors qu'elles ne font que désigner unilatéralement un aspect de la réalité, tout en masquant les aspects qui gênent ou font peur. D'où l'importance de réfléchir sur nos manières de sentir, de penser, d'agir, bref sur nos "épistémologies » implicites et nos attitudes profondes envers le monde qui nous entoure.

Albert Jacquard, dans son ouvrage L'éloge de la différence (Jacquard, 1978), rassemble ses réflexions finales sous le thème "l'amour des différences ». Partant d'un bel aphorisme de Saint-Exupéry, "Si je diffère de toi, loin de te léser, je t'augmente", Jacquard (1978: 206) fait une éloquente plaidoirie en faveur de la richesse de la différence. Tout en dénonçant ce «besoin superficiel de confort intellectuel qui nous pousse à tout ramener à des types et à tout juger selon la conformité aux types ", il rappelle le besoin fondamental que nous avons d'être unique pour "être " vraiment. N'est-il pas plus gratifiant en effet pour l'intelligence humaine de comprendre l'autre dans ce qu'il a d'unique, d'accueillir en nous le " cadeau » de sa différence, même si ce cadeau risque de nous déranger dans notre vision unilatérale ou binaire des choses?

Quel plus beau cadeau peut nous faire l' «autre» que de renforcer notre unicité, notre originalité, en étant différent de nous? II ne s'agit pas d'édulcorer les conflits, de gommer les oppositions; mais d'admettre que ces conflits, ces oppositions doivent et peuvent être bénéfiques à tous (Jacquard, 1978: 206207). 
Bien que les réflexions de Jacquard soient basées principalement sur ses observations dans le domaine de la génétique, il n'hésite pas à les transposer au domaine de la culture. Son éloge de la différence s'étend à l'ensemble des rapports humains à l'échelle des civilisations, qui sont merveilleusement diverses, et dont la diversité constitue la richesse de chacun de nous (Jacquard, 1978: 208). Sur cette piste, il est rejoint $d^{\prime}$ ailleurs par Y. Schwartz, qui consacre la première partie d'une volumineuse thèse aux relations complexes entre "cultures et incultures". Se plaçant d'emblée dans une perspective socio-historique, Schwartz remet en question l'idée d'une unification culturelle à l'échelle mondiale qui justifierait la disparition des différences culturelles dans le développement historique des sociétés.

Non seulement l'unification culturelle n'est pas un fait aujour$d^{\prime}$ hui mais on peut même s'interroger sur le sens d'une telle idée: I'histoire, sur la base de la production matérielle de leur vie par les groupes humains, ne s'arrête jamais, elle a produit des individus, des peuples et des civilisations aux héritages différents, et ces différences ne cessent de produire des effets moteurs en dehors desquels aucune intelligibilité de cette histoire n'est possible (Schwartz, 1988: 74).

Cette prise de position se situe aux antipodes d'un superficiel « relativisme culturel », dans la mesure où, par-delà la juxtaposition ou la coexistence entre des cultures diverses dans une société de type melting pot, elle préconise le vrai respect des singularités sur la base de l'expérience universelle des hommes et des femmes dans la production de leur vie sociale. II y a à travers des formes essentielles de "concentrations " humaines, souligne l'auteur en s'appuyant sur l'anthropologue André Leroi-Ghourhan, des facteurs différentiels mais contraignants de connexion entre leurs développements (Schwartz, 1988: 75).

En somme, ce qu'il est primordial de souligner ici, $c^{\prime}$ est le caractère indissociable de deux tendances inhérentes à tout individu, à tout groupe et à toute société en développement, selon une dialectique profonde de I'universel et du particulier dans le singulier: d'une part, l'identité se différencie dans un processus de division interne pour actualiser toutes ses potentialités et ses configurations singulières; $d$ 'autre part, la différence cherche à se dépasser dans un universel plus riche et plus concret.

\section{ÉTHIQUE DE LA DIFFÉRENCE}

L'acceptation ou le rejet de la différence est une question d'éthique et de politique. En effet, ceux qui interviennent auprès des minorités raciales, ethniques et culturelles se trouvent toujours à la «croisée des chemins » 
(Christensen et al., 1991), au point de contact culturel entre personnes, groupes, communautés, associations ou organismes qui ne sont pas au départ nécessairement sur la même longueur $\mathrm{d}^{\prime}$ onde.

Selon Bateson (cité dans Miermont, 1987: 465), trois issues sont possibles au contact culturel entre deux groupes: la fusion complète des deux groupes initialement différents, l'élimination d'un des groupes, voire des deux, la persistance des deux groupes initiaux, qui maintiennent une interface entre eux et établissent alors un équilibre dynamique à l'intérieur d'une communauté plus grande qui les réunit. De toute évidence, l'issue la plus souhaitable serait celle d'un équilibre dynamique entre les groupes. Équilibre maintenu non pas au prix du sacrifice des identités, mais à la faveur d'une différenciation réciproque des identités.

La différence reconnue et travaillée est porteuse d'une unité plus riche, plus dynamique, plus conviviale, la différence niée et évacuée tend à se transformer en antagonismes insurmontables. Ainsi en est-il des différences de sexe, d'âge, de culture, de race, d'ethnie, de classe, d'idéologies, de convictions politiques et de croyances religieuses, qui constituent autant de médiations par lesquelles le social nous est accessible. Dans les périodes de crise, la tension entre le besoin d'affirmer son identité et la nécessité de respecter l'autre devient insoutenable, et les médiations s'estompent devant les préjugés, les malentendus, les phénomènes $d^{\prime}$ ostracisme et de racisme, d'exclusion et de réclusion, de violence et de discrimination ${ }^{2}$.

La société québécoise, qui peut à juste titre se féliciter d'avoir réussi son passage à la modernité, commence à s'interroger sur un certain nombre de problèmes dont les manifestations étaient difficilement visibles durant les années de la "grande noirceur». C'est comme si la nouvelle société en gestation depuis la révolution tranquille se découvre aujourd'hui, en pleine maturité, devant des enjeux et des défis auxquels elle ne croyait pas devoir faire face comme d'autres sociétés dont l'histoire a été marquée par les luttes interraciales, interethniques et interculturelles.

Le Québec, particulièrement dans la région métropolitaine de Montréal, est traversé par de nouveaux types de rapports sociaux entre ethnies, races et cultures différentes. Il y a une tendance très répandue à définir ces rapports exclusivement en termes de conflits entre les "communautés culturelles" et la "communauté d'accueil», au risque de masquer les différences et les contradictions réelles à l'intérieur de chaque communauté. Ces mutations socioculturelles constituent, à n'en pas douter, un signe de maturation et d'enrichissement. Toutefois, elles suscitent aussi des questions éthiques importantes pour les formateurs-chercheurs, intervenants et étudiants qui $s^{\prime}$ intéressent de près aux problèmes sociaux liés à l'immigration. Car les immigrants, qu'ils soient réfugiés, nouveaux 
arrivants ou résidents au Québec depuis plusieurs années, sont partie prenante de ces mutations socioculturelles et les vivent parfois à travers des situations critiques dans un contexte économique marqué par la récession, le chômage et l'accroissement dramatique de la pauvreté. II existe d'ailleurs dans l'opinion publique québécoise une certaine tendance à attribuer principalement ces problèmes économiques à la présence plus ou moins "visible» des immigrants, lesquels sont souvent stigmatisés comme "voleurs de job», "fauteurs de troubles», "délinquants ». Ces préjugés sont amplifiés par la désinformation et la distorsion qui caractérisent la façon dont certains médias couvrent les événements impliquant des personnes ou groupes appartenant aux différentes minorités raciales, culturelles ou ethniques.

Quelles que soient les définitions qu'on leur donne, les problèmes vécus par les immigrants sont réels et ont des répercussions multiples sur les plans personnel, familial et social. Ces problèmes devraient faire l'objet $d^{\prime}$ 'une attention particulière, sans toutefois être isolés de leur contexte sociohistorique et politique. Or, les politiques québécoises en matière d'emploi et d'immigration, d'éducation et de culture, de services sociaux et de santé $\mathrm{n}^{\prime}$ ont pas vraiment préparé la population et les institutions à faire face à cette soudaine explosion de problèmes interraciaux, interethniques et interculturels. Les récentes campagnes de sensibilisation et les efforts faits dans le cadre des services publics et parapublics pour faciliter I'accueil, la formation, l'embauche et l'intégration culturelle des immigrants sont certes encourageants, mais loin d'être suffisants pour prévenir et stopper les manifestations violentes de conflits dans les rapports interethniques, interculturels et interraciaux. Montréal, comme Toronto, Los Angeles, New York, a été secouée au cours des dernières années par des confrontations violentes et meurtrières entre forces policières et communautés noires, ou entre groupes extrémistes de toutes tendances (dont les Skin Heads et le Ku-Klux-Klan) et "étrangers».

II y a un manque flagrant de ressources matérielles et humaines pour faire face aux problèmes vécus par les immigrants en tenant compte à la fois de la spécificité de la situation immigrante et de l'ensemble des rapports sociaux au sein d'une société changeante. Les intervenants sociaux travaillant dans les établissements de services sociaux et dans les organismes communautaires ne sont pas toujours suffisamment outillés pour faire face à cette nouvelle problématique. D'où la nécessité de se pencher sur les besoins en formation, tant à l'université que sur le terrain, pour tous ceux qui sont appelés à intervenir dans ce domaine.

La formation donnée jusqu'à maintenant par les unités de formation universitaire en travail social est axée principalement sur des conceptions et des modèles d'intervention qui n'ont pas été révisés de manière à 
intégrer une réflexion en profondeur sur l'intervention dans le contexte des nouveaux rapports interculturels, interethniques et interraciaux. II y a, par contre, une prise de conscience très nette, chez beaucoup de formateurs-chercheurs, d'étudiants et d'intervenants sur le terrain, de la nécessité de renouveler les théories, les méthodes et les techniques d'intervention dans ce contexte. Cette prise de conscience coïncide avec une conjoncture favorable à l'introduction de changements significatifs dans les programmes et les politiques institutionnelles, dans chacune des unités de formation en travail social au Québec. Il s'agit là d'un pas dans la bonne direction sur une route toutefois encore minée par de très nombreuses idées préconçues, un foisonnement de modèles improvisés et de multiples ambiguiités dans la manière d'aborder les relations interraciales, interethniques et interculturelles. Selon nous, ces relations seront vécues de manière d'autant plus gratifiante et stimulante que les personnes et les groupes reconnaîtront et accepteront leurs différences comme une source d'enrichissement mutuel plutôt que comme une source de problèmes ou de conflits. Cela implique la construction de nouvelles solidarités par la négociation coopérative des différences entre individus, familles, groupes et communautés qui se côtoient dans une société en mutation.

\section{CADRE POUR UNE NÉGOCIATION COOPÉRATIVE DES DIFFÉRENCES}

Au départ de toute entreprise de négociation qui se fait selon les règles, il y a présomption de bonne foi de la part des partenaires, même si ces derniers argumentent à partir de positions différentes. Présomption aussi que chacun a la capacité et la volonté de rechercher la vérité à partir de certaines normes reconnues et acceptées par tous. Toutefois, la vérité ne se laisse jamais posséder comme une "chose" univoque et absolue. Toujours concrète et militante, elle se laisse plutôt chercher à travers de multiples contradictions et dans un long processus d'assimilation critique. Le texte suivant de Gramsci à propos de la discussion scientifique offre, selon moi, quelques éléments intéressants qui peuvent servir de cadre pour l'élaboration d'un modèle de négociation coopérative des différences.

Dans la manière de poser les problèmes historico-critiques, il ne faut pas concevoir la discussion scientifique comme un procès de justice où il y aurait un accusé et un procureur, qui doit de par sa fonction démontrer que l'accusé est coupable et qu'il mérite d'être retiré de la circulation. Dans une discussion scientifique, vu que l'on suppose que l'intérêt réside dans la recherche de la vérité et le progrès de la science, celui qui se place du point de vue selon lequel l'adversaire peut exprimer une exigence qui doit être intégrée, même comme moment secondaire, à sa propre construc- 
tion, s'avère être le plus "avancé ". Comprendre et évaluer de manière réaliste les positions et les raisons de l'adversaire (et parfois toute la pensée passée est un adversaire) signifie justement se libérer du carcan des idéologies (au sens péjoratif de fanatisme aveugle). C'est se placer d'un point de vue « critique», le seul qui soit fécond dans la recherche scientifique (Gramsci, 1978: 67-68).

Ce texte fait essentiellement l'éloge du jugement critique, comme pivot de la discussion scientifique. Il est construit en quatre propositions, dont les trois premières sont interreliées sous la forme d'un syllogisme. La première proposition énonce négativement un principe général dont la force réside dans le rejet catégorique d'une conception judiciariste de la discussion scientifique: le rapport entre les partenaires d'une discussion $n^{\prime}$ en est pas un d'exclusion mutuelle entre accusateur et accusé sous I'autorité morale d'un juge habilité à trancher la question en litige. La seconde proposition définit positivement la nature de ce rapport: il s'agit $d^{\prime}$ une coopération entre des adversaires, des sujets porteurs de points de vue opposés, qui acceptent de les confronter sur la base de leur intérêt commun pour la vérité et le progrès de la science. Au lieu de faire appel à la médiation externe d'un juge, les partenaires conviennent de rester dans la circulation pour vérifier, en interface, la validité, la justesse et la force de leurs propres jugements critiques. La médiation de l'exigence universelle de recherche de la vérité se fait par l'intérêt particulier que chaque partenaire entretient pour sa part de vérité, et cette part de vérité, chacun la construit dans un double rapport d'identification et de différenciation avec le point de vue de l'autre. La troisième proposition énonce de manière synthétique la conséquence des deux premières propositions: la discussion scientifique est essentiellement un processus de compréhension et d'évaluation des positions et des raisons de l'adversaire. Ce processus d'intercompréhension n'est possible que si chaque partenaire se libère du carcan des idéologies qui empêche de voir et de juger correctement. La dernière proposition ramasse dans une formulation lapidaire la quintessence du texte: pour aborder la recherche scientifique le seul point de vue fécond est le point de vue critique.

Plusieurs auteurs contemporains, connaisseurs ou ignorants de l'œuvre de Gramsci, ont systématisé une réflexion sur l'éthique de la discussion à partir de principes semblables à ceux énoncés dans le texte cité plus haut. Ce qui vaut pour la discussion scientifique peut, à des nuances près, s'étendre à toute discussion qui réunit autour d'une table des partenaires recherchant la vérité et la justice à partir d'expériences et de connaissances différentes, mais selon une exigence morale universelle, étrangère à tout fanatisme et à tout dogmatisme. C'est la thématique que propose, entre autres, Jürgen Habermas dans ses recherches en collaboration avec Apel et Gadamer. Conscient des critiques soulevées par son 
ouvrage Morale et communication, Habermas (1986) fait le point sur les fondements de "l'éthique de la discussion", en cohérence d'ailleurs avec sa «théorie de l'agir communicationnel ».

On comprendra que le cadre du présent article ne permette pas d'entrer dans les détails du débat entre différentes écoles de pensée sur l'éthique, la morale et la déontologie. II nous suffit de marquer au passage certaines observations de Habermas sur la place des différences dans une éthique de la discussion, observations qui nous semblent tout à fait pertinentes pour renouveler la question éthique dans la formation en travail social.

L'auteur souligne les avantages d'aborder la question éthique en accordant une prépondérance au point de vue du «juste» sur celui du "bien », et en partant de la procédure de la discussion pratique. Car, «dans les argumentations, les participants doivent partir du fait qu'en principe tous les concernés prennent part, libres et égaux, à une recherche coopérative de la vérité dans laquelle seule peut valoir la force sans contrainte du meilleur argument» (Habermas, 1992: 19-20). Ce principe a des conséquences importantes pour la formation intellectuelle et morale des intervenants, en particulier en ce qui a trait à leur agir communicationnel, comme partie intégrante de leur pratique sociale: "la discussion pratique est considérée comme une forme exigeante de formation argumentative de la volonté qui doit garantir par les seules présuppositions universelles de la communication la justesse (l'équité) de tout accord normatif possible conclu dans ces conditions».

Même si « la discussion pratique se laisse concevoir comme un processus d'intercompréhension » qui se déroule dans le cadre d'une "opération publique pratiquée par tous intersubjectivement en commun ", elle ne peut faire abstraction des interactions sociales qui lient entre eux des sujets différents dans une société concrète. En effet, plus les principes d'égalité s'imposent à la praxis sociale, plus les formes et les projets de vie se différencient les uns des autres. Et plus cette multiplicité est grande, plus abstraite doit être la forme que doivent adopter les règles et les principes qui protègent l'intégrité et l'égal droit à la coexistence de sujets et d'êtres vivants devenant de plus en plus étrangers les uns aux autres, et ne démordant pas de leur différence et altérité (Habermas, 1992: 179).

Les intervenants sociaux, qui font des interactions sociales la matière première de leur pratique, font face dans leur formation comme sur le terrain à la tension paradoxale entre le respect de l'intégrité des personnes et les besoins de protection de ces personnes sur une base collective à cause de leurs vulnérabilités réciproques.

Les interactions sociales qui relèvent d'un usage du langage orienté vers l'intercompréhension sont constitutives des formes de 
vie socioculturelle. Ce type de socialisation communicationnelle, par laquelle, en même temps, chacun est individué, fonde une profonde vulnérabilité, parce que l'identité des individus socialisés ne se développe que par la voie d'une intégration au sein de dépendances sociales toujours plus grandes. La personne ne forme un centre intérieur, ne vient à elle-même, que dans la mesure où elle s'aliène également à des relations interpersonnelles produites communicationnellement, et s'entremêle à un filet toujours dense et subtil de vulnérabilités réciproques et de besoins de protection... Personne ne peut affirmer son intégrité pour lui tout seul. L'intégrité des personnes individuelles exige la stabilisation d'une réseau de rapports de reconnaissance symétriques, au sein desquels des individus insubstituables ne peuvent assurer leur fragile identité en tant que membres d'une communauté que réciproquement (Habermas, 1992: 196).

Ce texte comporte en filigrane les orientations d'un programme de formation à la négociation coopérative des différences dans I'intervention ethnoculturelle. Le code d'éthique du travail social, dans sa formulation américaine, canadienne ou québécoise, stipule le respect de l'intégrité et de la dignité personnelles, l'amélioration des conditions sociales des individus, groupes et communautés desservis, et ce sans discrimination de race, de couleur, de religion, d'âge, de sexe, d'origine ethnique ou culturelle ${ }^{3}$. Toutefois, pour interpréter et évaluer les multiples contradictions inhérentes aux interactions sociales entre sujets appartenant à des races, ethnies et cultures différentes, la formation en travail social devrait offrir à l'intervenant, par-delà le code d'éthique professionnelle, les outils nécessaires pour expérimenter et développer une praxis sociale fondée sur la " compréhension modificatrice» (Tosel, 1984: 281 et s.). Cette approche des situations même les plus conflictuelles par la compréhension modificatrice est une des conditions requises pour constituer un réseau stable de "rapports de reconnaissance symétriques" entre des individus insubstituables en tant que membres d'une communauté. C'est aussi la voie proposée par Henri Atlan dans ses considérations sur l'éthique de l'éducation pour faire "pousser la vérité de terre " par un dialogue axé sur la recherche d'un terrain de compromis et de consensus:

$C^{\prime}$ est seulement d'un dialogue avec recherche de compromis entre cultures et traditions différentes qu'on peut espérer que naisse, par construction progressive et de façon empirique, sans révélation imposée ni menace d'excommunication, une universalité fondée sur la reconnaissance d'autrui (Atlan, 1991 : 274).

L'apprentissage de cette compréhension modificatrice consiste dans l'acquisition d'un ensemble de capacités sans doute déjà visées par la formation en travail social, mais qui demandent à être constamment renforcées et raffinées en vue de renouveler l'intervention en contexte 
multiracial, multiethnique et multiculturel. Il s'agit des capacités 1 ) de sentir les implications des différences raciales, ethniques et culturelles présentes dans notre monde vécu ici et maintenant et dans le monde social (espaces publics, institutions juridico-politiques, organismes socioculturels, établissements de services sociaux et de santé), 2) de comprendre la complexité de ces différences en tenant compte des niveaux multiples auxquels elles se manifestent en nous et autour de nous, au niveau microscopique ou infinitésimal comme au niveau macroscopique ou systémique, 3) et d'intégrer les savoirs particuliers acquis à partir de ces différences, sur la base de la pratique clinique, dans une conception critique du monde leur permettant de s'engager en connaissance de cause dans les processus de transformation sociale. L'acquisition de ces capacités s'inscrit dans un processus circulaire que Gramsci, dans une note célèbre des Cahiers de prison à propos des rapports organiques entre les intellectuels et les masses populaires, définit comme un "passage du savoir au comprendre, au sentir, et vice versa du sentir au comprendre, au savoir » (Gramsci, 1978: 299). Il s'agit, selon nous, d'une des meilleures avenues éthico-politiques à expérimenter pour renouveler la praxis sociale ${ }^{4}$.

\section{CONCLUSION}

Notre réflexion sur l'éthique de la différence en travail social s'inscrit, croyons-nous, dans l'important renouveau de la question éthique au vingtième siècle. Renouveau particulièrement marquant en cette fin de siècle troublée par les nombreuses discussions et argumentations contradictoires concernant les progrès de la biologie et les nouvelles technologies médicales, I'avortement, l'écologie, l'euthanasie, I'usage généralisé des drogues, le sida, la paix dans le monde, l'informatisation et les droits de la personne, la crise de l'État et de ses appareils d'hégémonie à l'échelle mondiale, etc. Tout cela a occasionné un chambardement des valeurs traditionnelles et une redéfinition des paramètres de l'action individuelle et collective. Poser un acte professionnel dans ce contexte sociohistorique aussi mouvementé équivaut, pour le praticien du social, à exercer l'art du funambule. Marcher sur une corde raide sans faillir, cela prend un sens de l'équilibre exceptionnel. C'est cet équilibre, jamais acquis une fois pour toutes, que nous sommes appelés à retrouver ou à renforcer pour nous orienter dans une société pluraliste, multiraciale, multiethnique et multiculturelle. Nous espérons avoir montré que cet équilibre dépend principalement de notre capacité de rechercher la vérité par la négociation coopérative des différences dans nos rapports multiples à l'autre qui est à la fois en nous, en face de nous et autour de nous. 


\section{Notes}

1. Compte tenu de la pratique courante d'interchanger les termes éthique, morale et déontologie, la définition suivante proposée par S. Auroux (1990:870) en se basant sur l'étymologie du terme éthique (ethos) constitue un bon point de repère : " Le mot éthique, initialement appliqué à la morale sous toutes ses formes, désigne plus spécialement : 1) l'étude théorique des principes qui guident l'action humaine dans les contextes où le choix est possible ; 2 ) tout ensemble de principes gouvernant l'action des individus pour autant qu'ils agissent en fonction de leur appartenance à un groupe social déterminé et que cette appartenance impose des règles de conduite. "

2. À ce propos, l'article de l'Encyclopedia universalis sous le terme Race souligne les conséquences désastreuses de certaines attitudes qui dérivent d'une négation fondamentale de la différence. Toutes les formes de discrimination, à l'échelle des relations internationales ainsi qu'au niveau des relations inter-groupes ou interpersonnelles, se ramènent à une fausse identification / assimilation de l'autre à soi pour l'opprimer, le réprimer et, à la limite, supprimer son existence.

3. La formulation du code d'éthique de la NASW est encore plus explicite sur la discrimination: "I will not discriminate because of race, color, religion, age, sex, or national ancestry, and in my job capacity will work to prevent and eliminate social discrimination in rendering services, work assignments, and in employment practices " (cité dans Compton and Galaway, 1975: 117). Il ne s'agit donc pas seulement de s'abstenir de tout acte discriminatoire, mais bien de prévenir et d'éliminer toute discrimination dans l'exercice de la profession à tous les niveaux.

4. La réfléxion théorique proposée ici a émergé au cours d'une recherche sur les liens entre la formation en travail social et les "communautés culturelles" dans la région montréalaise. Cette même proposition sert de fil conducteur pour la réalisation d'un projet de laboratoire de recherche et d'intervention sur les différences raciales, ethniques et culturelles.

\section{Références bibliographiques}

ATLAN, Henri (1991). Tout, non, peut-être. Éducation et vérité. Paris : Seuil.

AUROUX, S. (1990). Encyclopédie philosophique universelle, tome 1. Paris : PUF, p. 870 .

BATESON, G. (1977). Vers une écologie de l'esprit. Paris : Seuil.

BATESON, G. (1984). La nature et la pensée. Paris: Seuil.

CHRISTENSEN, C. et al. (1991). L'enseignement du service social à la croisée des chemins: le défi de la diversité. Rapport d'enquête présenté au ministère du Multiculturalisme et de la Citoyenneté canadienne par un groupe de travail de l'Association canadienne des écoles de service social (ACESS) sur la place des questions multiraciales, multiethniques et multiculturelles dans la formation donnée par les écoles de service social agréées par I'ACESS.

Compton, B.R. et B. Galaway (1975). Social Work Processes. Homewood, Ill. : The Dorsey Press.

Cossom, J. (1992). "What Do We Know About Social Workers' Ethics? ", Le travailleur social, vol. 60, $\mathrm{n}^{\circ} 3$ : 165-171.

Encyclopedia universalis. Voir le terme Race. 
FÉdération NATIONALE dES COMITÉS D'ENTENTE ET DE LIAISON DES CENTRES DE FORMATION DES TRAVAILLEURS SOCIAUX (1988). La théorie et le savoir dans l'acte du praticien. Dijon.

GRAMSCI, A. (1975). Quaderni del carcere. Torino: Einaudi Editore.

GRAMSCI, A. (1978). Cahiers de prison, vol. III. Paris: Gallimard.

GRAMSCI, A. (1983). Cahiers de prison, vol. II. Paris: Gallimard,.

HABERMAS, Jürgen (1986). Morale et communication, traduit de l'allemand par Christian Bouchindhomme. Paris: Éditions du Cerf.

HABERMAS, Jürgen (1987). Théorie de l'agir communicationnel, 2 tomes, traduit de l'allemand par Jean-Marc Ferry. Paris: Fayard.

HABERMAS, Jürgen (1992). De l'éthique de la discussion, traduit de l'allemand par Mark Hunyadi. Paris : Éditions du Cerf.

HeGEL, G.W.F. (1991). Phénoménologie de l'Esprit, traduit de l'allemand par JeanPierre Lefevbre. Paris : Aubier.

JACQUARD, Albert (1978). Éloge de la différence. La génétique et les hommes. Paris : Seuil. Coll. Points.

JOUTHE, Ernst (1992). «Approfondir les différences dans la formation en travail social », Études doctorales dans un contexte de redéfinition du social. Actes de la journée du doctorat en service social tenue le 22 novembre 1991, École de service social, Faculté des sciences sociales, Université Laval.

MieRMONT, Jacques (1987). Dictionnaire des thérapies familiales. Paris: Payot.

Relations (1991). Numéro 571.

RHODES, Margaret L. (1986.). Ethical Dilemmas in Social Work Practice. Londres: Routledge \& Kegan Paul.

SCHWARTZ, Yves (1988). Expérience et connaissance du travail. Paris: Messidor. Service social (1991). Volume 40, numéro 1.

TOSEL, André (1984). Praxis. Paris : Éditions sociales. 